\title{
Role of Image-defined risk factors in clinical practice of neuroblastoma: A systematic review and meta-analysis
}

\author{
Qiyang Shen ${ }^{1}$, Qi Han ${ }^{1}$, Hanjun $\mathrm{Yin}^{2}$, Li Lu ${ }^{3}$, Tao Li ${ }^{1}$, and Jianfeng Zhou ${ }^{1}$ \\ ${ }^{1}$ Children's Hospital of Nanjing Medical University \\ ${ }^{2}$ Suqian People's Hospital of Nanjing Drum-Tower Hospital Group \\ ${ }^{3}$ Jiangsu Provincial Hospital of Chinese Medicine
}

June 22, 2020

\begin{abstract}
Purpose: To assess the role of image-defined risk factors in clinical practice of neuroblastoma. Methods: A systematic search was conducted to identify articles about IDRFs. Combined overall results were calculated using fixed effects models. Results: The literature search identified 11 articles including 1338 patients for analysis. There was no difference in amplification of MYCN and tumor site (abdominal tumor) between no IDRFs and any IDRFs group, but there was a significant increase of event free survival (OR: 2.43[1.69, 3.49]; $\mathrm{P}<0.00001$ ) and overall survival (OR: $3.15[1.68,5.89]$; $\mathrm{P}=0.0004)$ in no IDRFs group. The incidence of complications (OR: $0.21[0.15,0.30] ; \mathrm{P}<0.00001)$ was higher in patients with any IDRFs. Furthermore, no IDRFs are correlated with higher rate of earlier INSS stage. Conclusions: IDRFs could be an important adjuvant image monitoring method in NB treatment. IDRFs are valuable to evaluate surgical indications for pediatric surgeon, regardless of surgical ability.
\end{abstract}

\section{Hosted file}

TitlePage.doc available at https://authorea.com/users/335737/articles/461544-role-of-imagedefined-risk-factors-in-clinical-practice-of-neuroblastoma-a-systematic-review-and-metaanalysis

\section{Hosted file}

manuscript.docx available at https://authorea.com/users/335737/articles/461544-role-ofimage-defined-risk-factors-in-clinical-practice-of-neuroblastoma-a-systematic-reviewand-meta-analysis 


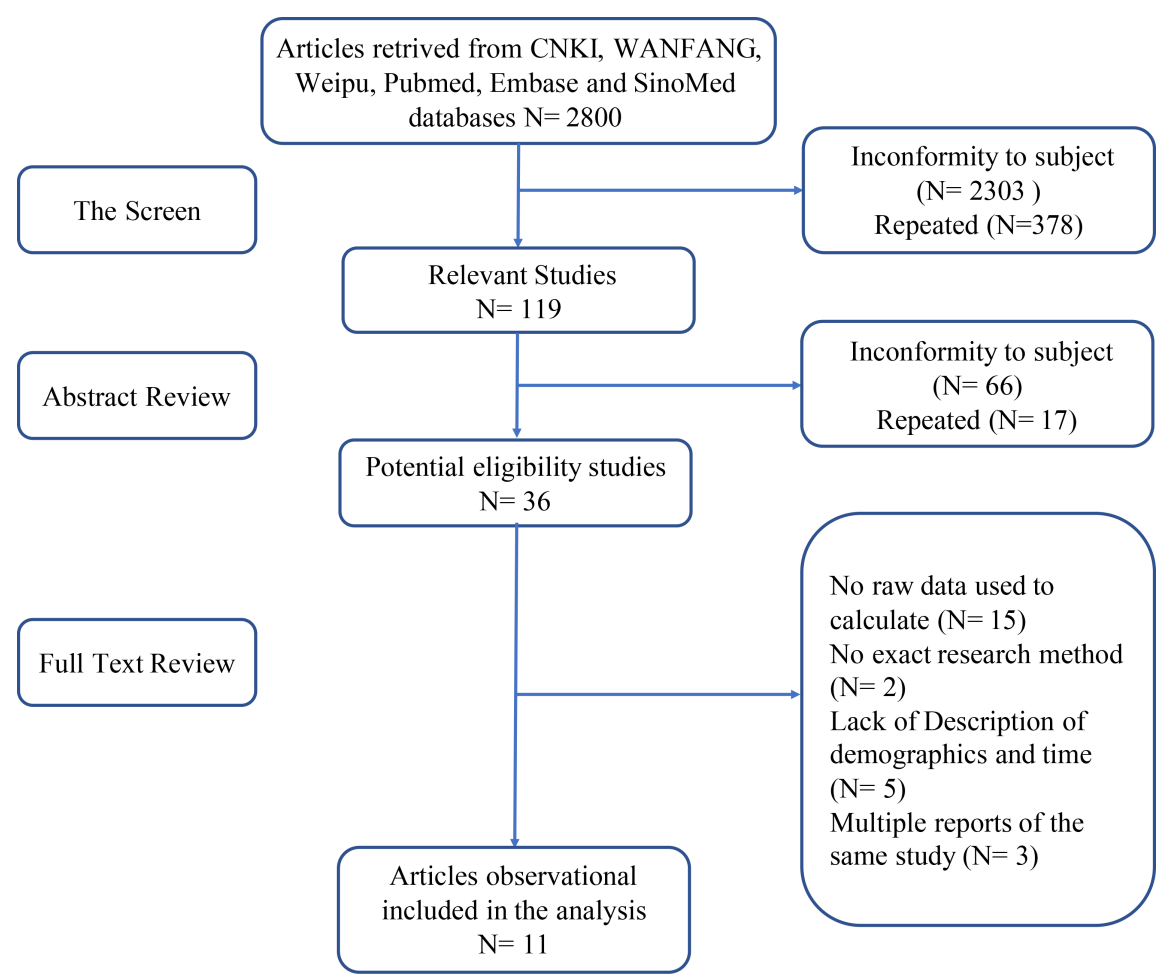



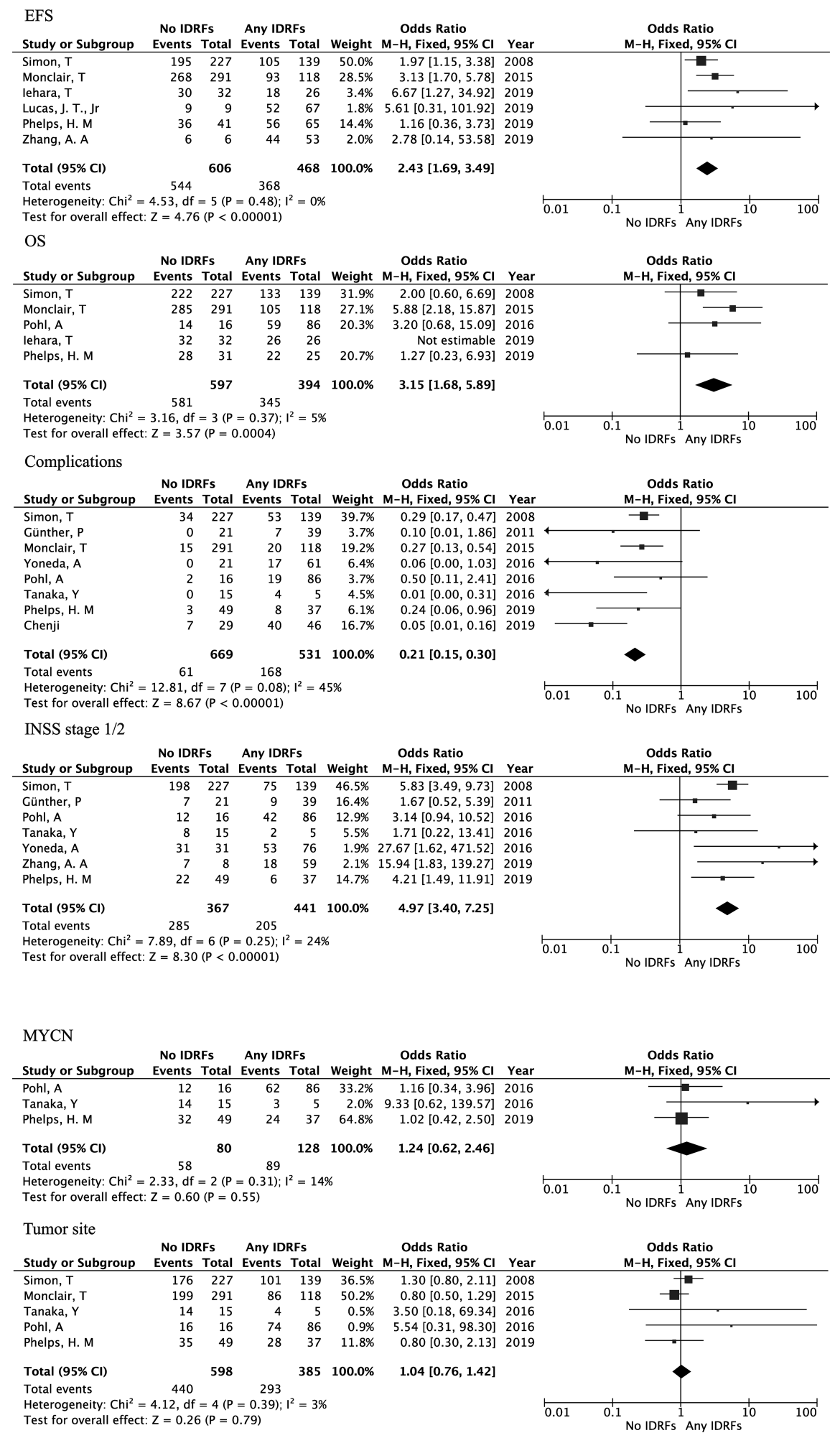

\section{Hosted file}

Table 1.docx available at https://authorea.com/users/335737/articles/461544-role-of-image- 
defined-risk-factors-in-clinical-practice-of-neuroblastoma-a-systematic-review-and-metaanalysis
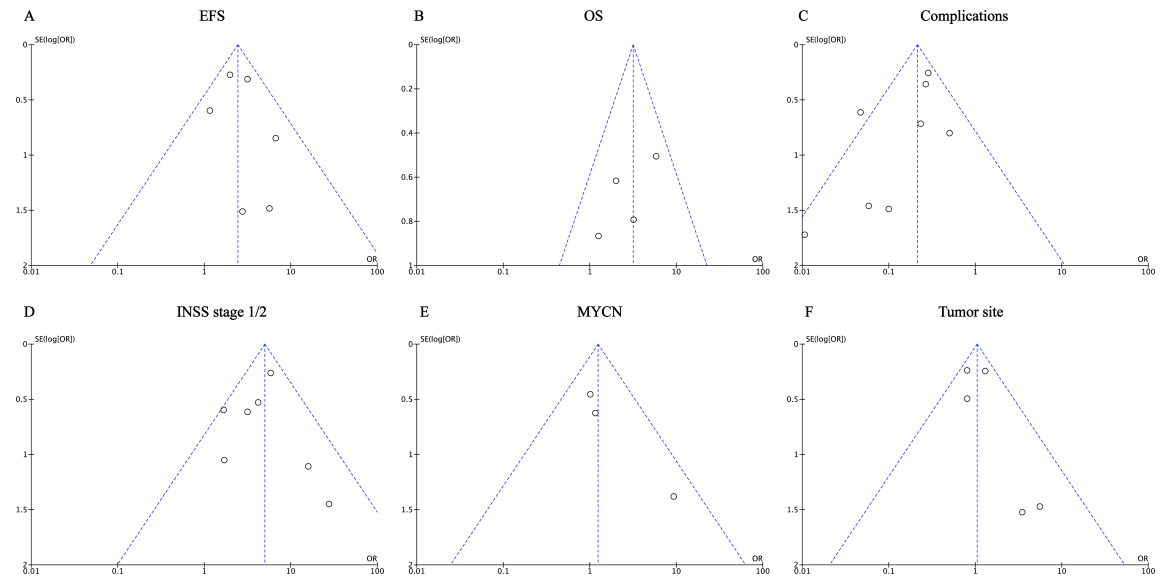
Zhang, A. A.2019

Phelps, H. M.2019

Lucas, J. T., Jr.2019

Iehara, T.2019

Yoneda, A.2016

Tanaka, Y.2016

Pohl, A.2016

Monclair, T.2015

Günther, P.2011

Simon, T.2008

\begin{tabular}{|c|c|c|c|c|c|c|c|}
\hline 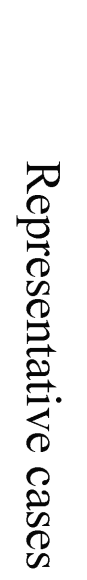 & 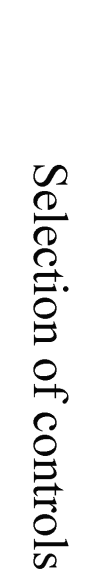 & $\begin{array}{l}\vec{D} \\
0 \\
0 \\
0 \\
0.0 \\
0.0 \\
0 \\
0 \\
0 \\
0 \\
0 \\
0 \\
0 \\
0 \\
0 \\
0 \\
0 \\
0\end{array}$ & 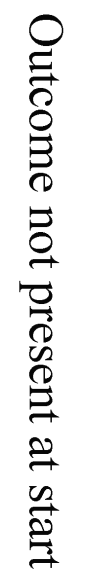 & 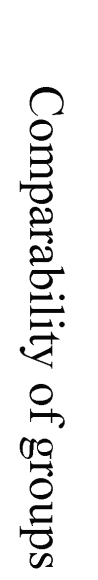 & 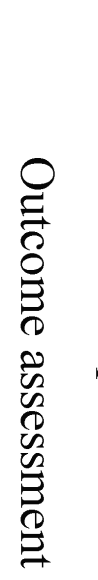 & 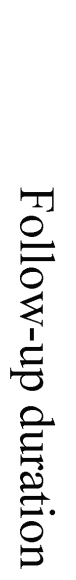 & 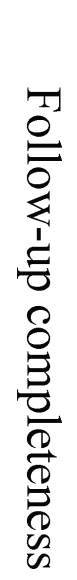 \\
\hline 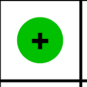 & $\oplus$ & 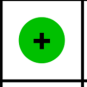 & $\odot$ & 0 & 0 & $\oplus$ & 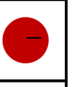 \\
\hline+ & + & + & + & + & & + & + \\
\hline$\odot$ & + & + & + & $\oplus$ & & + & $\theta$ \\
\hline+ & $\oplus$ & $\oplus$ & $\odot$ & $\oplus$ & & 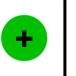 & + \\
\hline 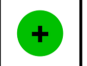 & $\oplus$ & $\oplus$ & + & $\oplus$ & & 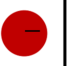 & $\odot$ \\
\hline+ & $\odot$ & $\oplus$ & $\odot$ & $\oplus$ & & 0 & 0 \\
\hline+ & $\odot$ & $\odot$ & $\odot$ & $\odot$ & & + & + \\
\hline$\oplus$ & + & + & $\odot$ & $\oplus$ & & $\oplus$ & + \\
\hline 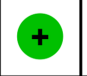 & $\odot$ & $\oplus$ & + & + & & ○ & $\ominus$ \\
\hline$\oplus$ & $\odot$ & $\oplus$ & $\odot$ & + & & $\oplus$ & + \\
\hline
\end{tabular}

